\title{
Simplified models for exotic BSM searches
}

\author{
Jan Heisig, ${ }^{a}$ Andre Lessa ${ }^{b, c}$ and Loic Quertenmont ${ }^{d}$ \\ ${ }^{a}$ Institute for Theoretical Particle Physics and Cosmology, RWTH Aachen University, \\ Sommerfeldstr. 16, 52056 Aachen, Germany \\ ${ }^{b}$ Instituto de Física, Universidade de São Paulo, \\ R. do Matão 187, São Paulo, SP 05508-900, Brazil \\ ${ }^{c}$ Universidade Federal do $A B C$, \\ Av. dos Estados, 5001, Santo André, SP 09210-580, Brazil \\ ${ }^{d}$ Centre for Particle Physics and Phenomenology, CP3, Université Catholique de Louvain, \\ Chemin du Cyclotron 2, B-1348 Louvain-la-Neuve, Belgium \\ E-mail: heisig@physik.rwth-aachen.de, andre.lessa@ufabc.edu.br, \\ loic.quertenmont@gmail.com
}

ABSTRACT: Simplified models are a successful way of interpreting current LHC searches for models beyond the standard model (BSM). So far simplified models have focused on topologies featuring a missing transverse energy (MET) signature. However, in some BSM theories other, more exotic, signatures occur. If a charged particle becomes longlived on collider time scales - as it is the case in parts of the SUSY parameter space it leads to a very distinct signature. We present an extension of the computer package SModelS which includes simplified models for heavy stable charged particles (HSCP). As a physical application we investigate the CMSSM stau co-annihilation strip containing long-lived staus, which presents a potential solution to the Lithium problem. Applying both MET and HSCP constraints we show that, for low values of $\tan \beta$, all this region of parameter space either violates Dark Matter constraints or is excluded by LHC searches.

KEYwords: Supersymmetry Phenomenology, Phenomenological Models

ARXIV EPRINT: 1509.00473 


\section{Contents}

1 Introduction 1

2 Simplified models for HSCPs 3

2.1 Overview of the CMS search for HSCP 3

2.2 Computation of signal efficiencies 5

$\begin{array}{lll}2.3 \text { Validation } & 7\end{array}$

$\begin{array}{lll}2.4 & \text { Results for the efficiency maps } & 7\end{array}$

3 Using simplified models to constrain full models $\quad 9$

4 Application to the Lithium-7 problem $\quad 12$

$\begin{array}{lll}4.1 & \text { Scan results } & 14\end{array}$

$\begin{array}{lll}4.2 & \text { Comparison with full simulation } & 18\end{array}$

$\begin{array}{lll}5 & \text { Conclusions } & 19\end{array}$

\section{Introduction}

In theories beyond the standard model (BSM) with an unbroken $\mathbb{Z}_{2}$-symmetry the lightest $\mathbb{Z}_{2}$-odd particle (LOP) is stable and hence usually required to be neutral as there exist strong bounds on the presence of stable charged particles in the universe [1-3]. The typical collider signature of such a BSM scenario is missing transverse energy (MET) caused by the invisible LOP escaping the collider. However, there are scenarios where a heavy $\mathbb{Z}_{2^{-}}$ odd particle can become sufficiently long-lived to appear as stable in a collider experiment. This particle can be charged and thus produce a very distinct signature.

Heavy stable charged particles (or HSCP) can appear when the next-to-lightest $\mathbb{Z}_{2^{-}}$ odd particle is nearly mass degenerate to the LOP and hence its decay is kinematically suppressed. A prominent example for such a situation is a supersymmetric scenario where a wino- or higgsino-like neutralino is the lightest supersymmetric particle (LSP). The typically small mass splitting between the lightest neutralino and the lightest chargino in such a scenario can render the chargino long-lived, see e.g. [4]. A similar situation might occur in models of extra dimensions [5]. Other supersymmetric scenarios with mass degenerate sparticles have been proposed in order to address the discrepancy between the ${ }^{6} \mathrm{Li}$, ${ }^{7} \mathrm{Li}$ abundances predicted in standard big bang nucleosynthesis (BBN) and those inferred from astrophysical observations [6]. In this scenario a bino-like neutralino and the lightest charged slepton are close in mass, resulting in a long-lived slepton [7]. Another possible scenario leading to HSCPs corresponds to the case where the LOP does not share the SM gauge couplings and only interacts super weakly. An important example are supersymmetric scenarios where the gravitino or axino is the LSP $[8,9]$. In this case the couplings are 
suppressed by powers of the Planck scale or the Peccei-Quinn scale and the life-time of the next-to-LSP (NLSP) can easily exceed the typical time for passing the detectors by many orders of magnitude.

Collider constraints on HSCPs have been mostly presented in specific BSM models [10$12]$ and in most cases cannot be directly applied to other BSM scenarios. Currently, Simplified Model Spectra (SMS) [13-16] have become a popular alternative for presenting less model-dependent constraints, which can then be systematically applied to specific BSM scenarios. The CMS and ATLAS collaborations typically interpret their results in terms of simplified models and methods to use these interpretations in a systematical way have been made publicly available for missing energy (MET) topologies by tools such as SMoDELS [17] and FASTLIM [18]. As a result it is now possible (under some approximations) to test general BSM scenarios with MET signatures against LHC data. So far a similar approach has not been considered for HSCP or mixed MET-HSCP scenarios, although it has been argued $[19,20]$ that SMS are particularly suitable for parametrizing the LHC sensitivity to HSCP signatures, since these are rather inclusive and depend almost only on the kinematics of the HSCP itself.

In this study we examine limits on the particle spectrum of BSM theories containing HSCPs making use of simplified models. For this purpose we introduce a set of eight simplified model topologies containing either one or two HSCPs in the final states and compute efficiencies for several values of the BSM masses appearing in each topology. The resulting efficiency grids or efficiency maps allows us to re-interpret previous results in a wide range of BSM models. In order to do this in a more general framework we incorporate these efficiency maps to the program package SModelS [17, 21]. Since the public version of SMODELS already contains a large number of LHC constraints on simplified models containing MET signatures, our modified version allows us to simultaneously apply both MET and HSCP constraints to full BSM models. Hence we are able to test scenarios where HSCP and MET constraints compete. As we will show, current LHC searches provide a high sensitivity to HSCPs $[11,12]$ and the HSCP signature can support an exclusion or discovery even if its contribution to the total BSM signal is subdominant.

As a HSCP lead to a non-standard signature that is not supported by common fast detector simulations particular attention has to be drawn to a reliable implementation of such an analysis. In this study we use a novel approach for the computation of signal efficiencies presented in ref. [11]. This method uses a parametrization of the CMS detector response as a function of the kinematic properties of the HSCP and allows us to accurately compute the CMS signal efficiencies for arbitrary models.

As an application of the SMS framework to HSCPs, we consider the stau co-annihilation strip in the Constrained Minimal Supersymmetric Standard Model (CMSSM). We focus on the nearly mass degenerate neutralino LSP and stau NLSP, which has been proposed in order to solve the ${ }^{7} \mathrm{Li}$ problem [22]. Using our extension of SMODELS, we study the implications of both MET and HSCP searches and show that all of the parameter space (with $\tan \beta=10$ ) consistent with a potential solution to the ${ }^{7} \mathrm{Li}$ problem and the observed Dark Matter relic abundance is excluded by either HSCP or MET constraints (or both). This region of the CMSSM parameter space has also been studied in [23, 24], although 
not focussing on the region of interest for the solution of the ${ }^{7} \mathrm{Li}$ problem. However, in [24] no particular attention was drawn to the derivation of the efficiencies for the HSCP signal and as an approximation the cross section limits from the inclusive stau production presented in [10] were used. By applying the SMS framework to the considered slice of the CMSSM our derivation of the HSCP constraints provide a significant improvement to the previous work.

The remainder of this paper is organized as follows. In section 2 we will define the simplified models used in our results and present the computation of efficiency maps as well as their validation against the results presented in the CMS search [11]. In section 3 we explain our implementation of the decomposition of a full BSM model into simplified model topologies and how these are used to constrain the full model. An application of our results to a CMSSM scenario with a nearly mass degenerate neutralino and stau will be presented in section 4 . We conclude in section 5 .

\section{Simplified models for HSCPs}

In this section we will briefly review how CMS searched for HSCPs as it has a direct impact on some of the choices that we make in this paper. We will then introduce a number of simplified models that contain one or two HSCPs in the final state. These models correspond to the simplest topologies and appear in several BSM theories. The models are summarized in table 1 (two HSCPs in the final state) and table 2 (one HSCP and one neutral BSM particle in the final state). For computing the efficiencies as a function of the topologies and the BSM masses appearing in the cascade decays, we must choose a specific BSM model for production and decay of the $\mathbb{Z}_{2}$-odd particles. Here we use the supersymmetric (SUSY) simplified models, which are listed in the last column of tables 1 and 2. In these, the HSCP is either the lightest chargino, $\widetilde{\chi}_{1}^{ \pm}$, or the lighter stau, $\widetilde{\tau}_{1}$. As part of the Simplified Model approximations, we assume that the efficiencies are weakly dependent on the spin of the HSCP.

\subsection{Overview of the CMS search for HSCP}

Heavy stable charged particles are highly penetrating particles that are expected to cross the entire CMS detector and reach the muon system, ${ }^{1}$ and are therefore experimentally reconstructed and identified as muon particles. However, because of their large mass and the limited energy available in LHC collisions, they will be travelling through the detector with a velocity $(\beta)$ significantly slower than the speed-of-light. Consequently, they will have an anomalously high ionization energy loss $(\mathrm{d} E / \mathrm{d} x)$ and a longer time-of-flight (TOF) than relativistic standard model particles. In the CMS search for HSCP [10], the CMS silicon tracker is used to measure the particle $\mathrm{d} E / \mathrm{d} x$, while the CMS muon system is used to measure the particle's TOF. The events are mostly selected online by a muon $\left(p_{\mathrm{T}}>45 \mathrm{GeV}\right)$ trigger. However, the trigger becomes inefficient when the particle velocity is too low $(\beta<0.45)$ due to the too long delay $(>25 \mathrm{~ns})$ for the particle to reach the muon

\footnotetext{
${ }^{1}$ The behavior of color-charged HSCPs is more complex [10], but we focus only on the case of lepton-like HSCPs in this paper.
} 


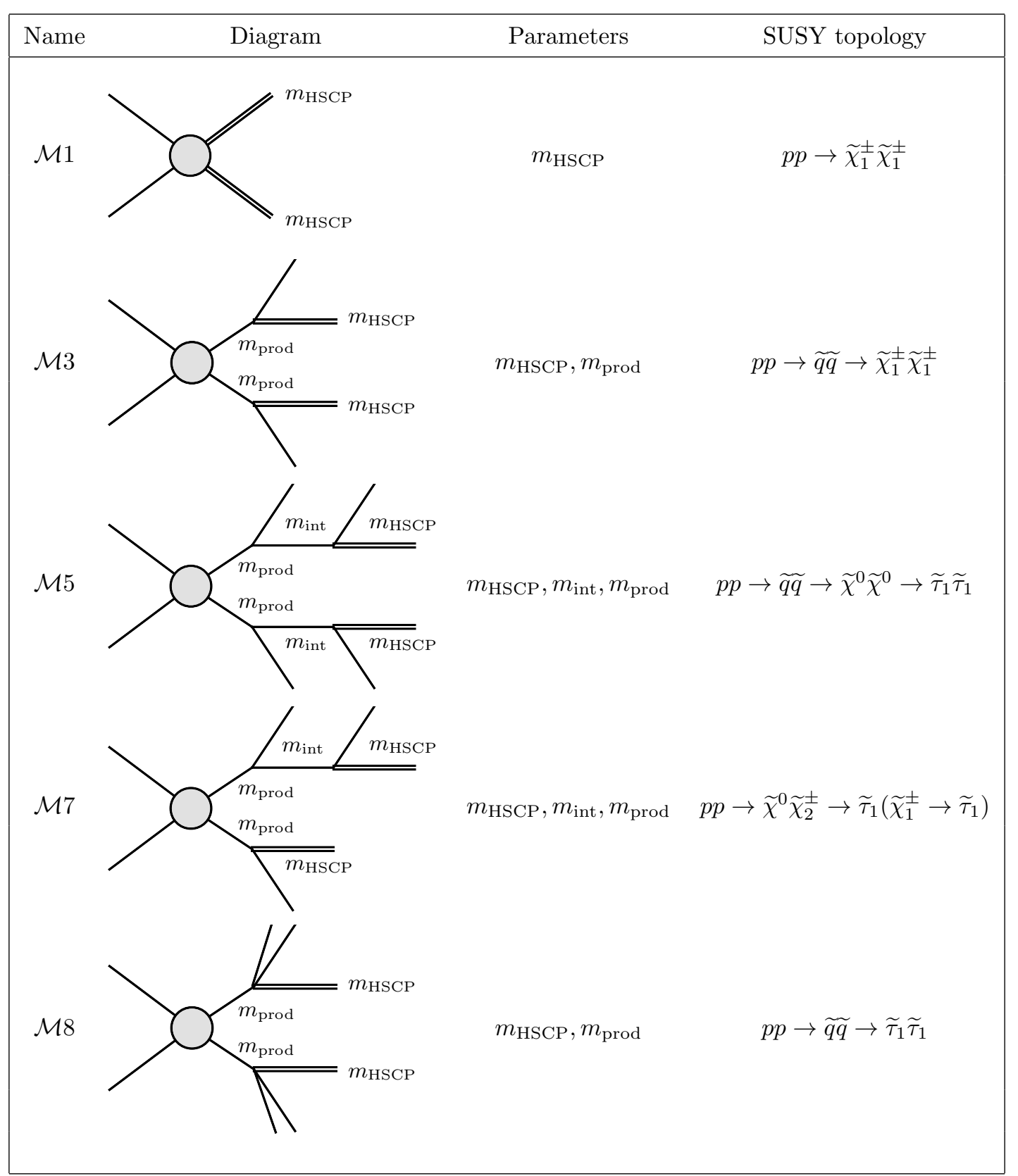

Table 1. Definitions of the simplified models with two HSCPs used in this study. In the diagrams single solid lines denote SM particles or intermediate BSM particles, double solid lines denote the HSCP.

system causing a mismatch between the muon system information and the inner tracker information.

While there is no real standard model background to this search, instrumental backgrounds due to the mis-measurement of either $\mathrm{d} E / \mathrm{d} x$ or TOF is not negligible. To predict the amount of backgrounds in the signal region CMS exploits the fact that the $\mathrm{d} E / \mathrm{d} x$ and TOF measurements are uncorrelated for backgrounds. The track $\mathrm{d} E / \mathrm{d} x$ and momentum 


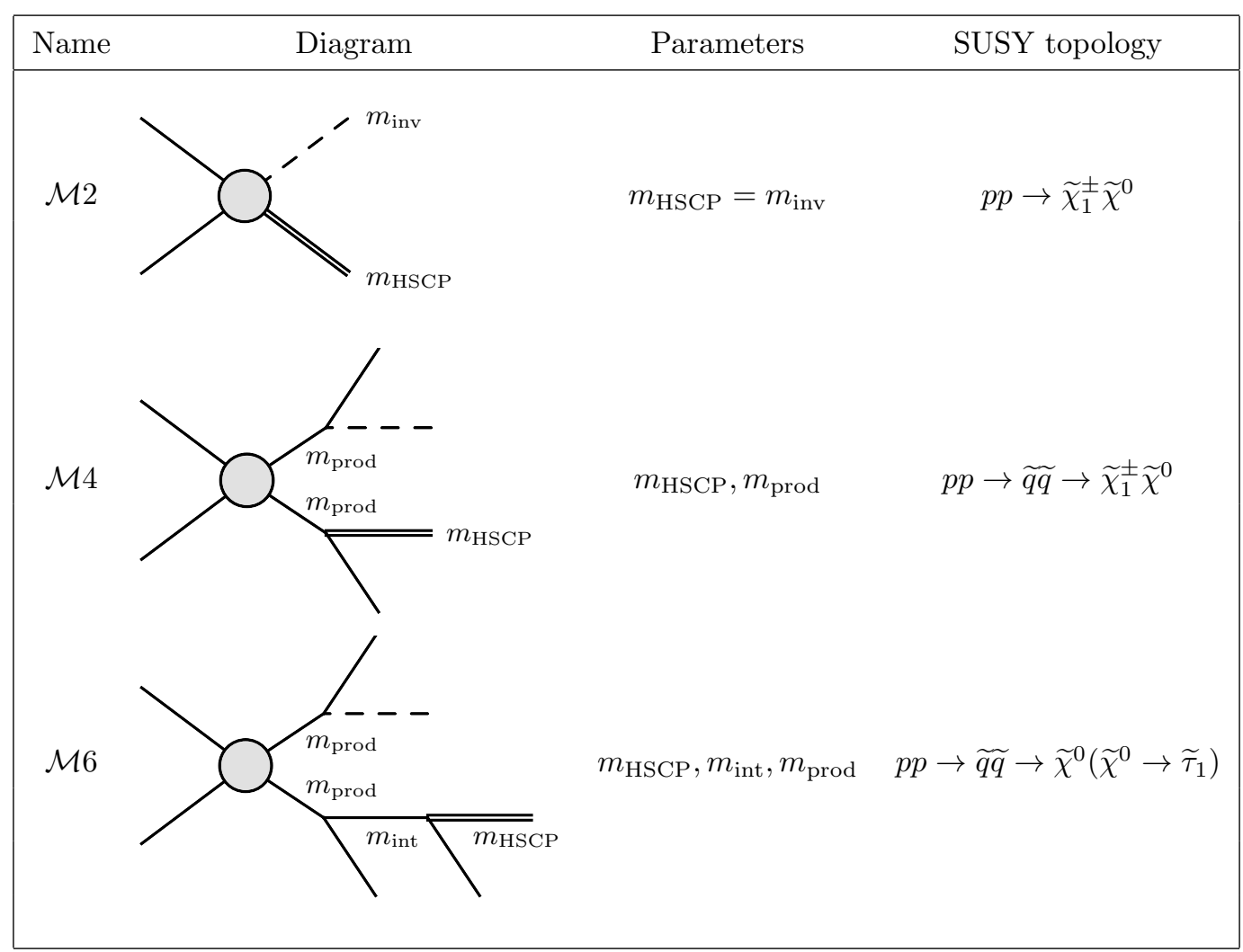

Table 2. Definitions of the simplified models with one HSCP used in this study. In the diagrams single solid lines denote SM particles or intermediate BSM particles, double solid lines denote the HSCP and dashed lines denote invisible particles (or an invisible branch, see figure 5).

variables are used to reconstruct the particle mass and further discriminate the HSCP signal from mis-reconstruction background peaking at low values of the reconstructed mass. Although the mass threshold used in [10] is continuous, the required inputs for the reinterpretation of these results [11] are only provided in $100 \mathrm{GeV}$ steps. Below we use the results presented in ref. [11] to compute the signal efficiencies for the simplified models introduced in tables 1 and 2 .

\subsection{Computation of signal efficiencies}

In order to compute the efficiencies for the simplified models, we perform a Monte Carlo simulation of the signal at the $8 \mathrm{TeV}$ LHC. For each topology listed in tables 1 and 2 we scan over the respective BSM masses (listed in the third column) and generate $30 \mathrm{k}$ events for each set of masses. For the event generation we use MADGRAPH 5 [25] to generate parton level events and then PyтніA 6 [26] to perform the decays, as well as showering and hadronization. No detector simulation is performed, since we follow the fast simulation procedure defined in ref. [11], where signal acceptances for HSCP candidates are provided as a function of the HSCP's kinematics. In order to identify HSCP candidates in each 
event we must first apply the following isolation criteria:

$$
\left(\sum_{j}^{\substack{\text { charged particles } \\ \Delta R<0.3}} p_{\mathrm{T}^{j}}\right)<50 \mathrm{GeV} \text { and }\left(\sum_{j}^{\substack{\Delta R<0.3 \\ \text { visible particles } \\|\boldsymbol{p}|}}\right)<0.3,
$$

where the first (second) sum includes all the charged (visible) particles in a cone of $\Delta R=\sqrt{\Delta \eta^{2}+\Delta \phi^{2}}<0.3$ around the direction of the long-lived particle, $p_{\mathrm{T}^{j}}$ denotes their transverse momenta, $E^{j}$ their energy and $|\boldsymbol{p}|$ is the magnitude of the long-lived particle's three-momentum. In both sums the long-lived particle candidate itself is not included. As muons release very little energy in the calorimeters they are not considered as visible particles. The purpose of these isolation requirements is to mimic the event selection used in the CMS analysis [10]. Long-lived particles failing any of these isolation requirements are not considered as HSCP candidates.

Once the HSCP candidates are identified, we can compute the signal efficiencies using the acceptances provided in ref. [11]. These acceptances are given as probabilities for the candidate to pass the on- and off-line selection criteria $\left(P_{\text {on }}\right.$ and $\left.P_{\text {off }}\right)$ and depend on the candidate's pseudo-rapidity $\eta$, transverse momentum $p_{\mathrm{T}}$ and velocity $\beta$. The final signal efficiency $(\epsilon)$ is then given by:

$$
\epsilon=\frac{1}{N} \sum_{i}^{N} P_{\text {on }}\left(\boldsymbol{k}_{i}\right) \times P_{\text {off }}\left(\boldsymbol{k}_{i}\right)
$$

where $P_{\text {on }}\left(P_{\text {off }}\right)$ is the on-line (off-line) probability for each event, the sum runs over all generated events, $N$, and $\boldsymbol{k}_{i}=\left(\eta_{i}, p_{\mathrm{T} i}, \beta_{i}\right)$ contains the kinematic properties for the HSCP candidate in the $i$ th event. For events containing two HSCP candidates, the above probabilities must be replaced by [11]

$$
P_{\text {on/off }}^{(2)}\left(\boldsymbol{k}_{i}^{1}, \boldsymbol{k}_{i}^{2}\right)=P_{\text {on/off }}\left(\boldsymbol{k}_{i}^{1}\right)+P_{\text {on/off }}\left(\boldsymbol{k}_{i}^{2}\right)-P_{\text {on/off }}\left(\boldsymbol{k}_{i}^{1}\right) P_{\text {on/off }}\left(\boldsymbol{k}_{i}^{2}\right),
$$

where $\boldsymbol{k}_{i}^{1,2}$ are the kinematical vectors of the HSCPs. There are two main effects governing $P_{\text {on/off }}$. On the one hand, the velocity $\beta$ should considerably deviate from 1 in order to allow for a discrimination against muons. Hence, for $\beta \rightarrow 1$ the acceptance goes to zero. On the other hand for too small $\beta(\beta \lesssim 0.45)$ the particle may not be assigned to the right bunch crossing anymore. In this case, the trigger efficiencies (online selection) go down very drastically.

The CMS analysis also requires a minimum reconstructed mass $\left(m_{\mathrm{rec}}\right)$ for the candidate. For the fast simulation method used here, the collaboration provides the $P_{\text {on/off }}$ probabilities for four distinct mass cuts, which we consider as four different signal regions:

$$
\begin{aligned}
& \mathrm{SR}_{0}: m_{\mathrm{rec}}>0 \mathrm{GeV}, \quad \mathrm{SR}_{100}: m_{\mathrm{rec}}>100 \mathrm{GeV} \text {, } \\
& \mathrm{SR}_{200}: m_{\mathrm{rec}}>200 \mathrm{GeV} \text { and } \mathrm{SR}_{300}: m_{\mathrm{rec}}>300 \mathrm{GeV} \text {. }
\end{aligned}
$$

Due to detector resolution effects, the reconstructed mass is typically $m_{\mathrm{rec}} \simeq 0.6 m_{\mathrm{HSCP}}[11]$ and the above requirements must be translated to the real HSCP mass. Therefore, when computing the efficiencies for each signal region, we take $\epsilon=0$, if $m_{\mathrm{HSCP}}<166 \mathrm{GeV}$, $334 \mathrm{GeV}$ and $500 \mathrm{GeV}$ for the signal regions $\mathrm{SR}_{100}, \mathrm{SR}_{200}$ and $\mathrm{SR}_{300}$, respectively. 

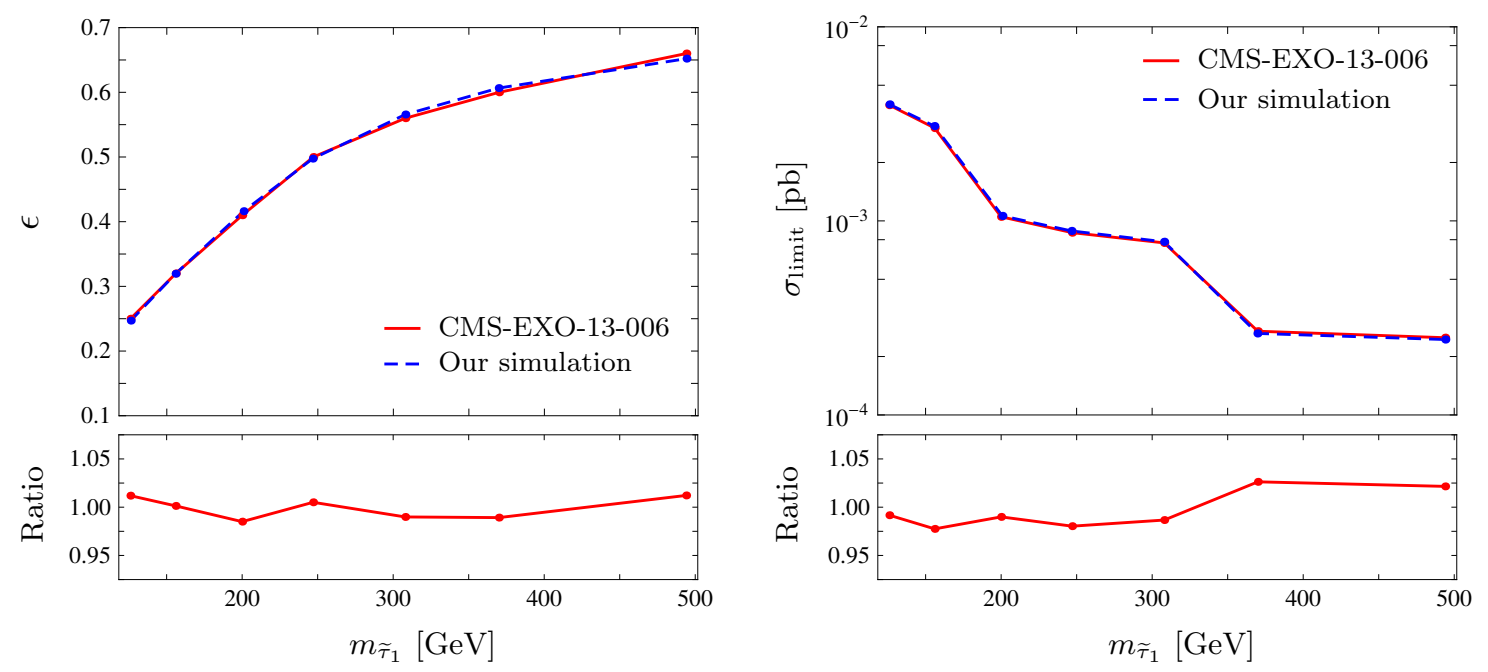

Figure 1. Signal efficiency $\epsilon$ (left panel) and 95\% CL cross section upper limit (right panel) for the GMSB model as the function of the stau mass. We compare the CMS analysis (CMS-EXO-13006 [11]) from the full detector simulation (red solid lines) with our implementation of the analysis described in section 2.2 (blue dashed lines). In the lower frames we show the respective ratios $\epsilon^{\mathrm{CMS}} / \epsilon^{\mathrm{Our}}, \sigma_{\text {limit }}^{\mathrm{CMS}} / \sigma_{\text {limit }}^{\text {Our }}$.

\section{$2.3 \quad$ Validation}

In order to validate the procedure described in section 2.2 , we compute the efficiencies for the gauge-mediated supersymmetry breaking (GMSB) models considered by the CMS collaboration in ref. [11]. These models have a gravitino LSP and a long-lived stau as the NLSP. As a result, for collider purposes, all the sparticles cascade decay to the lightest stau, which is the HSCP candidate. We simulated the signal with PyтнiA 6 and analyzed the generated events as described in section 2.2. The results obtained for the inclusive production of staus are shown in figure 1, where we also show the corresponding efficiencies obtained by the CMS collaboration. As in ref. [11], we choose $\mathrm{SR}_{0}$ for $m_{\mathrm{HSCP}}<166 \mathrm{GeV}$, $\mathrm{SR}_{100}$ for $166 \mathrm{GeV}<m_{\mathrm{HSCP}}<334 \mathrm{GeV}$ and $\mathrm{SR}_{200}$ for higher masses. Our efficiencies agree within $3 \%$ with the ones obtained by CMS, where the differences are likely due to Monte Carlo statistical uncertainties. Therefore our procedure for computing the signal efficiencies reproduce very well the experimental results and can be used to produce the efficiency maps for the simplified models listed in tables 1 and 2. In figure 1 we also reproduce the $95 \%$ CL limits on the inclusive production cross sections, which again agree very well (within $\sim 3 \%$ ) with the ones obtained by CMS from ref. [11]. Note that this limit is based on the discrete mass cuts on $m_{\text {rec }}$ mentioned above, the full CMS analysis allows for a event-based mass cut, resulting in somewhat stronger constraints for some values of the HSCP mass.

\subsection{Results for the efficiency maps}

Our procedure to compute the efficiency maps using the method outlined in section 2.2 is as follows. For the eight models listed in tables 1 and 2 we generate events and compute 


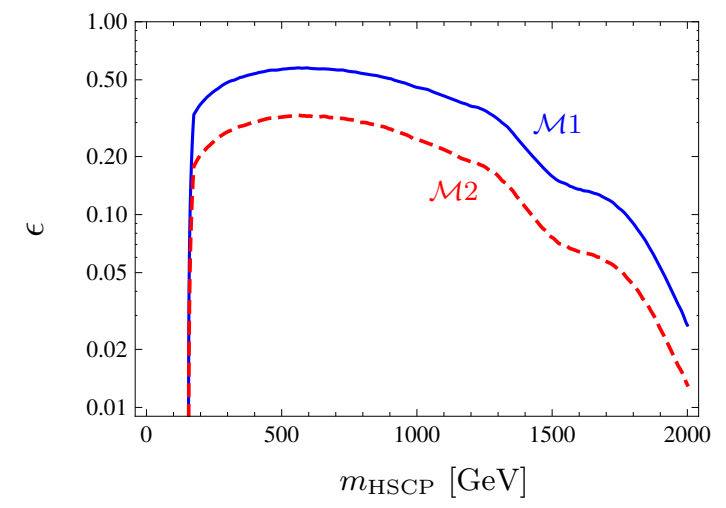

Figure 2. Signal efficiency $\epsilon$ as a function of the mass of the HSCP for model $\mathcal{M} 1$ (direct production of two HSCPs, blue solid curve) and model $\mathcal{M} 2$ (direct production of one HSCP and one invisible particle, red dashed curve).
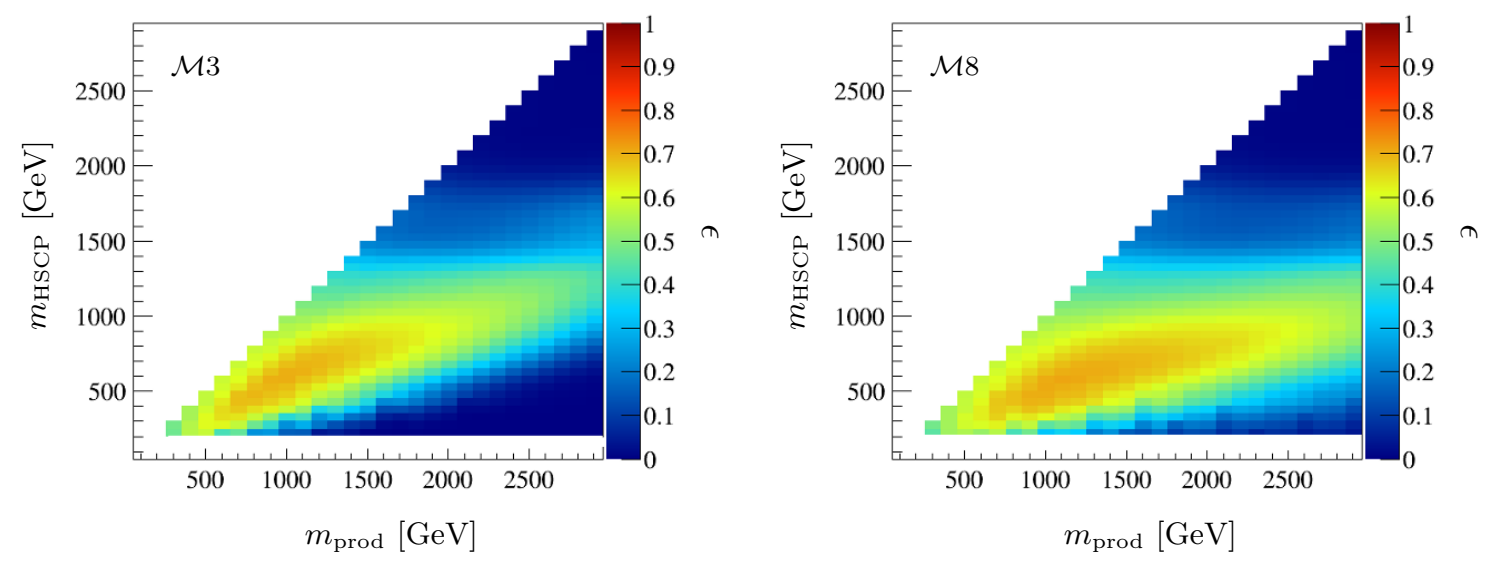

Figure 3. Signal efficiencies for model $\mathcal{M} 3$ (left panel) and model $\mathcal{M} 8$ (right panel).

the efficiencies in a wide range of sparticle masses between $50 \mathrm{GeV}$ and $3 \mathrm{TeV}$, varying the HSCP mass and all other masses listed in the tables in steps of $10 \mathrm{GeV}$ and $50 \mathrm{GeV}$, respectively. In order to allow for a fast processing within SMODELS we then reduce the number of mass points in regions of parameter space where the efficiencies do not vary considerably. As an example, we show in figures 2 and 3 the efficiencies for the $\mathrm{SR}_{100}$ signal region $\left(m_{\mathrm{rec}}>100 \mathrm{GeV}\right.$ or $\left.m_{\mathrm{HSCP}}>166 \mathrm{GeV}\right)$ for the simplified models $\mathcal{M} 1, \mathcal{M} 2$ and $\mathcal{M} 3, \mathcal{M} 8$, respectively.

The signal efficiencies for the models with two HSCPs can be as high as $70 \%$ and strongly depend on the HSCP mass. For direct production of two HSCPs (simplified model $\mathcal{M} 1$ ), the signal efficiency stays above $20 \%$ for masses between $166 \mathrm{GeV}$ and $1.4 \mathrm{TeV}$. As discussed in section 2.2, we take $\epsilon=0$ for $m_{\mathrm{HSCP}}<166 \mathrm{GeV}$ in the $\mathrm{SR}_{100}$ signal region. This is the reason for the sharp drop in the efficiencies in the light HSCP region, seen in figure 2. For very large masses the particles are produced very close to threshold, with extremely small $\beta(\lesssim 0.5)$ and the signal efficiency drops again. This is a result of the low detection probabilities (in particular the on-line probability) due to small trigger efficiencies for 
$\beta<0.5$. In models $\mathcal{M} 3$ and $\mathcal{M} 8$ the HSCPs are produced in the decay of heavier particles and the efficiencies are largest in the intermediate mass range $500 \mathrm{GeV}$ to $1.2 \mathrm{TeV}$. For large mass gaps between the produced particle and the HSCP, the latter becomes extremely boosted making the discrimination against the muon background extremely hard. Hence, the signal efficiency decreases rapidly in this region. This decrease is less pronounced in model $\mathcal{M} 8$, as the three-body decay leaves less energy to the HSCP.

As mentioned above, in the SMS framework used here we neglect sub-leading effects due to the spin of the HSCP or any other BSM particle. Nonetheless, we checked that, for direct production of the HSCP, the differences in the efficiencies are smaller than $20 \%$ when comparing the $s$-channel production of spin $0,1 / 2$ and 1 particles as well as the $t$-channel production of spin $1 / 2$ particles. For the production of BSM particles through longer decay chains we expect the differences to be even smaller, as the kinematics of the HSCP are more strongly influenced by the mass spectrum of the model. We hence expect that neglecting spin effects as well as the effects of the production channels ( $s$-channel versus $t$-channel) generates uncertainties of the order of $20 \%$ or below. Since these uncertainties are of the order of other theoretical uncertainties (such as NLO corrections to the sparticles production cross sections), we consider them acceptable.

\section{Using simplified models to constrain full models}

The efficiency maps described in section 2.4 allows us to compute the predicted signal cross section $\left(\sigma_{\mathrm{th}}\right)$ for a given simplified model $\mathcal{M}_{i}$ in one of the four signal regions $\left(\mathrm{SR}_{j}\right)$ :

$$
\left(\sigma_{\mathrm{th}}^{\mathcal{M}_{i}}\right)_{\mathrm{SR}_{j}}=\sigma^{\mathcal{M}_{i}} \times \epsilon_{\mathrm{SR}_{j}}^{\mathcal{M}_{i}}
$$

where $\sigma^{\mathcal{M}_{i}}$ is the cross section for the simplified model and $\epsilon_{\mathrm{SR}_{j}}^{\mathcal{M}_{i}}$ the respective efficiency for the signal region $\mathrm{SR}_{j}$. Comparing $\sigma_{\mathrm{th}}$ with the experimental upper limit for the signal cross section in the respective signal region $\left(\sigma_{\mathrm{UL}}\right)$, it is possible to determine if the simplified model is excluded or not by the experimental searches. However, simplified models rarely match any model of interest and their usefulness relies on the fact that, under some approximations, it is possible to decompose a full model in terms of a coherent sum of simplified models (see ref. [17] for details). In this case, the full model signal cross section $\left(\sigma_{\mathrm{th}}^{\text {Full }}\right)$ to be confronted with $\sigma_{\mathrm{UL}}$ is approximately given by:

$$
\left(\sigma_{\mathrm{th}}^{\text {Full }}\right)_{\mathrm{SR}_{j}}=\sum_{i} \tilde{\sigma}^{\mathcal{M}_{i}} \times \epsilon_{\mathrm{SR}_{j}}^{\mathcal{M}_{i}}
$$

In the above expression $\tilde{\sigma}^{\mathcal{M}_{i}}$ is the corresponding weight for the simplified model $\mathcal{M}_{i}$ in the full model. These weights are computed by the decomposition procedure, which maps the full model into a sum of simplified models topologies. Since the decomposition method used here follows closely the one used by SModeLS [17], we only outline the main steps, focusing on the differences required to treat long-lived particles.

First, using as input an SLHA card, all the widths $(\Gamma)$, branching ratios (BRs), masses and production cross sections $(\sigma)$ of the BSM states are defined for the input model. ${ }^{2}$

\footnotetext{
${ }^{2}$ In order to read the SLHA file, SModelS uses the tools provided by the PYSLHA [27] code.
} 
Second, for each of the particles appearing in the production cross sections, we generate all possible cascade decays, ${ }^{3}$ using the branching ratios and widths read from the SLHA card. However, since now the input model may contain quasi-stable states, we must determine if the particle at the end of the cascade decay is long-lived or not. More specifically, we must estimate what is the probability for a BSM state to have a prompt decay or to decay outside the detector. The fraction of particles which survive after traveling a distance $l$ in the detector is given by:

$$
f(l)=e^{-\Gamma l /(\gamma \beta)}
$$

where $\Gamma$ is the particle's width, $\gamma=1 / \sqrt{1-\beta^{2}}$ and $\beta$ is the particle's boost. Therefore, the probability for a particle to decay promptly is:

$$
\mathcal{F}_{\text {prompt }}=1-e^{-\Gamma l_{\text {inner }} /(\gamma \beta)},
$$

where $l_{\text {inner }}$ corresponds to the inner size of the detector, for which all decays are seen as prompt. For our subsequent results we take $l_{\text {inner }}=10 \mathrm{~mm}$. On the other hand, the probability for a particle to decay outside the detector is given by:

$$
\mathcal{F}_{\text {long }}=e^{-\Gamma l_{\text {outer }} /(\gamma \beta)},
$$

where $l_{\text {outer }}$ corresponds to the detector size, which we take to be $10 \mathrm{~m}$ (for CMS). Clearly the above probabilities are event-dependent, since they depend on the boost of the unstable particle, through the factor $\gamma \beta$. Nonetheless we can still conservatively estimate these. Since the efficiencies for a long-lived particle to be identified as a charged track fall sharply below $\beta \simeq 0.45$, here we take $(\gamma \beta)_{\text {outer }}=0.6$ (or $\beta \simeq 0.5$ ), which gives a mostly conservative estimate of $\mathcal{F}_{\text {prompt }}$. On the other hand, for prompt decays we take $(\gamma \beta)_{\text {inner }}=10$, which corresponds to $\beta \simeq 0.995$. Notice that for most models, $\Gamma$ is such that the particle can be considered as decaying promptly or long-lived for a wide range of $\gamma \beta$ values. Therefore, for most cases, our results are only mildly dependent on our choice of $\gamma \beta$.

Once $\mathcal{F}_{\text {prompt }}$ and $\mathcal{F}_{\text {long }}$ are known for each state, during the decomposition each unstable particle (with a non-zero width) will generate two possible topologies: ${ }^{4}$

- one where the particle does not decay (it is considered as long-lived). In this case the topology weight will be proportional to the probability for the particle to decay outside the detector $\left(\mathcal{F}_{\text {long }}\right)$;

- one where the particle decays promptly. In this case the topology weight will be proportional to the probability for the particle to decay inside the inner detector $\left(\mathcal{F}_{\text {prompt }}\right)$.

Notice that in most cases we have $\left(\mathcal{F}_{\text {long }}, \mathcal{F}_{\text {prompt }}\right) \simeq(1,0)$ for quasi-stable (or stable) particles or $\left(\mathcal{F}_{\text {long }}, \mathcal{F}_{\text {prompt }}\right) \simeq(0,1)$ for unstable particles.

\footnotetext{
${ }^{3}$ Since the total number of all possible cascade decays is typically of the order of hundreds of thousands, we neglect all topologies which have $\tilde{\sigma}^{\mathcal{M}_{i}}<\tilde{\sigma}_{\text {min }}$. In the results presented below we take $\tilde{\sigma}_{\text {min }}=5 \times 10^{-3} \mathrm{fb}$ $\left(5 \times 10^{-4} \mathrm{fb}\right)$ for the points with $m_{\mathrm{HSCP}} \leq 400 \mathrm{GeV}\left(m_{\mathrm{HSCP}}>400 \mathrm{GeV}\right)$.

${ }^{4}$ The case of displaced vertices is not considered in the present work.
} 


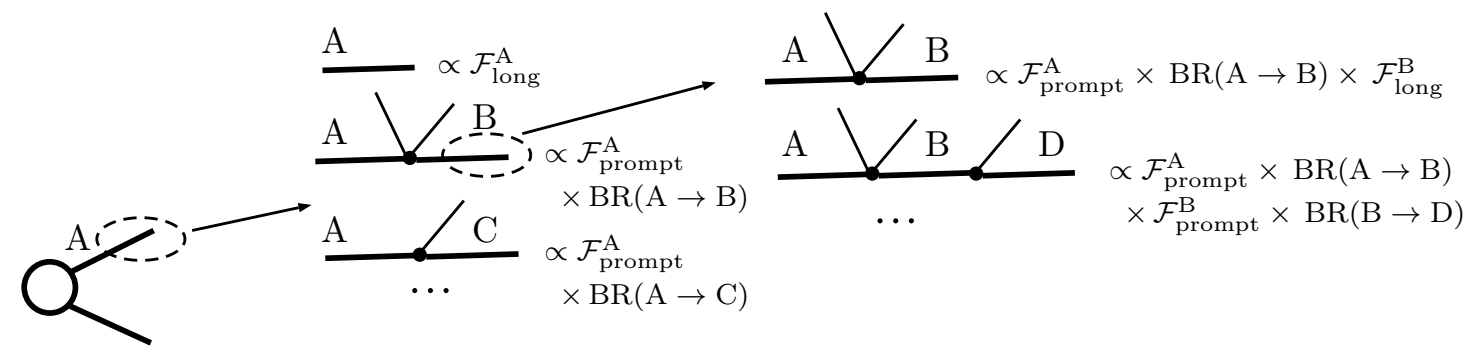

Figure 4. Decomposition procedure for scenarios containing quasi-stable particles. Next to the topologies we show the factors contributing to the topology weight. See text for details.

Using the information in the SLHA input and a modified version of the package SMODELS, which includes the computation of the $\mathcal{F}_{\text {prompt/long probabilities, it is possible to de- }}$ compose full models into distinct simplified model topologies and compute their weights. As mentioned above, the decomposition procedure starts with the pair production of BSM states (determined by the cross sections read from the SLHA card) and generates all possible cascade decays for each particle produced. For each step in the cascade decay, the topology weight is given by the product of the production cross section $\left(\sigma_{\text {prod }}\right)$, the BRs for the decays appearing in the decay chain and the prompt decay/long-lived fractions, $\mathcal{F}_{\text {prompt/long: }}$

$$
\tilde{\sigma}^{\mathcal{M}_{i}}=\sigma_{\text {prod }} \times\left(\prod_{i} \mathrm{BR}_{i} \times \mathcal{F}_{\text {prompt }}^{i}\right) \times \mathcal{F}_{\text {long }}^{X} \mathcal{F}_{\text {long }}^{Y},
$$

where $\mathcal{F}_{\text {long }}^{X} \mathcal{F}_{\text {long }}^{Y}$ is the product of the (non-decay) probabilities for the final states $(X, Y)$ appearing in the cascade decay chain. This procedure is outlined in figure 4. Since we only keep the topologies with a final weight above a minimum value $\left(\tilde{\sigma}_{\text {min }}\right)$, the topologies containing very small probabilities $\left(\mathcal{F}_{\text {prompt }} \ll 1\right.$ or $\left.\mathcal{F}_{\text {long }} \ll 1\right)$ are automatically discarded. This procedure has the advantage of allowing us to probe scenarios where more than one particle is (meta-)stable and to automatically determine which states can be considered as long-lived or decaying promptly. Furthermore, for models containing both neutral and charged (meta-)stable particles, the above procedure will produce topologies with both HSCP and missing energy (MET) signatures (or mixed MET-HSCP). Therefore it allows us to simultaneously confront the corresponding model with both MET and HSCP searches.

Once the topology weights $\left(\tilde{\sigma}^{\mathcal{M}_{i}}\right)$ are known, through eq. (3.2) it is possible to compute the full model signal cross section for each of the signal regions considered by the experimental searches. It is important to notice that, since we only computed efficiencies for the simplified models appearing in tables 1 and 2, the signal from any other topologies appearing during the decomposition procedure are not included in the final signal. Although this leads to conservative predictions, we will show below that, for the models studied here we only underestimate the signal cross section by $20 \%$ or less. At this point it is also relevant to stress that the current public version of SMODELS does not include efficiency maps for the MET searches. For these the topology weights cannot be summed up and the experimental upper limits for the individual $\tilde{\sigma}^{\mathcal{M}_{i}}$ weights are used instead (for 


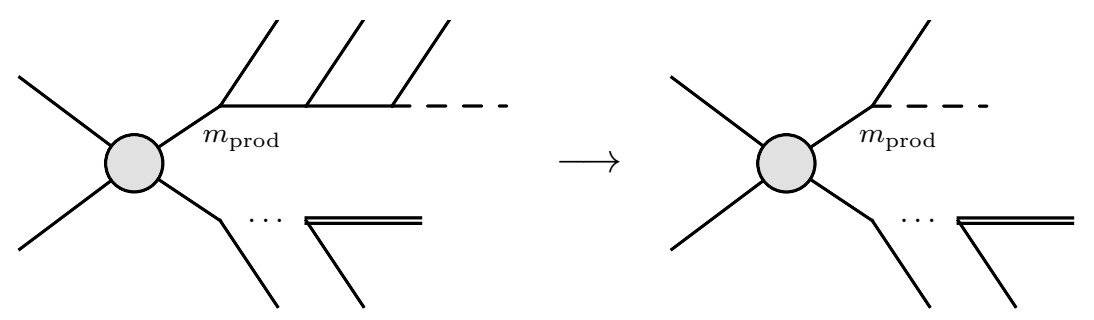

Figure 5. Models with one (arbitrarily long) decay chain terminating in an invisible particle are mapped onto the respective model with a single step decay in the invisible (MET) branch.

more details see ref. [17]). Since the decomposition procedure can produce topologies with one invisible and one HSCP in the final states (such as the ones shown in table 2), these can be constrained by both MET and HSCP searches. However, since HSCP constraints are typically stronger, the mixed topologies are considered as containing a single HSCP and we only apply the constraints from HSCP searches. Furthermore, since the HSCP efficiencies for these mixed topologies are almost independent of the cascade decay ending in the invisible state, we can neglect the kinematics of the MET branch and compress it to a single step decay, as shown in figure 5. This allows us to use the efficiencies computed for the simplified models $\mathcal{M}_{4}$ and $\mathcal{M}_{6}$ for a wide range of HSCP-MET topologies and improve the coverage of our efficiency maps.

Using the efficiency maps for the simplified models $\mathcal{M} 1-\mathcal{M} 8$ computed in section 2.4 and the decomposition procedure described above, we proceed to apply our modified version of SModels to a physical model of interest. As we will show, both MET and HSCP searches can be relevant (although the former are typically stronger) and allows us to impose strong constraints on the stau co-annihilation region of the CMSSM.

\section{Application to the Lithium-7 problem}

In this section we apply the procedure outlined in section 3 to a full model containing long-lived particles. One interesting motivation for the existence of long-lived particles (in cosmological scales) is the Lithium-7 problem [28, 29] (for a recent review, see ref. [30]). Despite the enormous success of BBN, the Lithium abundance inferred from the Cosmic Microwave Background and BBN [30],

$$
\left(\frac{{ }^{7} \mathrm{Li}}{\mathrm{H}}\right)_{\text {theo }}=(4.68 \pm 0.67) \times 10^{-10},
$$

is highly inconsistent with the experimentally measured Lithium abundance [31]:

$$
\left(\frac{\mathrm{Li}}{\mathrm{H}}\right)_{\exp }=(1.6 \pm 0.3) \times 10^{-10}
$$

Although some of the proposed solutions to the above discrepancy do not involve new physics (e.g. stellar depletion or inclusion of new nuclear reactions), these are usually 
highly tuned or require modification of nuclear rates well outside the expected uncertainties [32]. A popular alternative is to invoke new physics during BBN, which could explain a smaller Lithium production rate (or an annihilation of the original Lithium abundance). A well studied scenario is supersymmetry with long-lived staus $(\widetilde{\tau})$. If $\widetilde{\tau}$ s are still present during BBN, they can form bound states with nuclei (such as ${ }^{7} \mathrm{Li}$ ) and deplete the Lithium abundance, thus providing a viable solution to the Lithium problem. Since such solutions have been discussed at length in the literature [6,22, 33-43], here we concentrate on their features relevant for the LHC searches.

In this work we focus on the case of a neutralino LSP and consider the CMSSM, closely following the discussion presented in ref. [22]. In order to cover the CMSSM parameter space we perform a Monte Carlo scan in the input parameters:

$$
m_{0}, M_{1 / 2}, A_{0}
$$

where $m_{0}$ is the universal soft scalar mass, $M_{1 / 2}$ is the universal soft gaugino mass and $A_{0}$ the trilinear soft term, all defined at the unification scale, $M_{\mathrm{GUT}} \simeq 2 \times 10^{16} \mathrm{GeV}$. We take the supersymmetric mass term $\mu$ to be positive $(\mu>0)$, while we fix the ratio of the Higgs vacuum expectation values to be $\tan \beta=10$. We also limit our results to negative values of $A_{0}$, since these enhance the radiative corrections to the Higgs mass. Similar results would be obtained for $\mu<0$ and $A_{0}>0$ except in this case we would have a larger discrepancy between the predicted and measured values for the anomalous magnetic moment of the muon $(g-2)_{\mu}$. The supersymmetric spectrum is generated with SPheno 3.2.1 [44], the sparticle production cross sections are computed using Pyтhia 6 and NLLfast [45-51] and the neutralino relic abundance is computed with MICROMEGAs 3.0.24 [52].

As computed in refs. $[6,41]$, in order to solve the Lithium problem, the stau yield and life-time must satisfy:

$$
Y_{\widetilde{\tau}}^{0} \gtrsim 10^{-13} \text { and } \tau_{\widetilde{\tau}} \gtrsim 1-100 \mathrm{~s}
$$

The latter condition requires the stau to be nearly degenerate with the LSP, which we assume to be the lightest neutralino. In particular, the mass difference $\delta m=m_{\widetilde{\tau}_{1}}-m_{\widetilde{\chi}_{1}^{0}}$ must be significantly smaller than the $\tau$ mass $(\delta m<0.1 \mathrm{GeV})$. In this quasi-degenerate scenario, the stau abundance before its decay is related to the neutralino relic abundance by [41]

$$
Y_{\widetilde{\tau}}^{0} \simeq \frac{Y_{\widetilde{\chi}^{0}}}{2\left(1+\mathrm{e}^{\delta m / T_{\mathrm{f}}}\right)}
$$

where $Y_{\widetilde{\tau}}^{0}$ is the stau yield after freeze-out (and before decay) and $Y_{\widetilde{\chi}^{0}}$ is the final neutralino yield (after staus have decayed), which can be obtained from its final relic abundance:

$$
Y_{\widetilde{\chi}^{0}}=\left(\frac{\Omega_{\widetilde{\chi}^{0}} h^{2}}{2.741 \times 10^{8}}\right)\left(\frac{\mathrm{GeV}}{m_{\widetilde{\chi}_{1}^{0}}}\right) .
$$

The neutralino freeze-out temperature, $T_{\mathrm{f}}$, can be well approximated by $T_{\mathrm{f}} \simeq m_{\widetilde{\chi}_{1}^{0}} / 25$ for the parameter space considered below. 
Before discussing the LHC constraints from MET and HSCP searches, we first impose the following set of minimal constraints to the CMSSM:

- a neutralino LSP;

- $\delta m=m_{\widetilde{\tau}_{1}}-m_{\widetilde{\chi}_{1}^{0}}<0.1 \mathrm{GeV}$;

- $Y_{\widetilde{\tau}}^{0}>10^{-13}$

- $120 \mathrm{GeV}<m_{h}<130 \mathrm{GeV} .^{5}$

Although we keep points with $\Omega_{\widetilde{\chi}^{0}} h^{2}>0.1289$ (which violate the Planck's $3 \sigma$ upper bound on the Dark Matter relic abundance [53]), we will explicitly identify in our results the region consistent with Planck.

Since the left-right mixing of staus is proportional to $A_{\tau}-\mu \tan \beta$, the stau mass not only depends on the scalar mass parameter $m_{0}$ but also strongly on $A_{0}$ and $\tan \beta$. On the other hand, the neutralino mass is mainly dependent on the gaugino mass parameter $M_{1 / 2}$. Therefore, the requirement $m_{\widetilde{\tau}_{1}} \simeq m_{\widetilde{\chi}_{1}^{0}}$ introduces a correlation between $M_{1 / 2}$ and $m_{0}, A_{0}, \tan \beta$. In particular, as $m_{0}$ increases (for a fixed $M_{1 / 2}$ value), $A_{0}$ must increase (in absolute value) in order to enhance the stau mixing and reduce its mass, which must satisfy $m_{\widetilde{\tau}_{1}} \simeq m_{\widetilde{\chi}_{1}^{0}} \propto M_{1 / 2}$. Therefore, whilst scanning over $A_{0}$ and $M_{1 / 2}$ with flat priors, we limit the scan over $m_{0}$ to a gaussian distribution around the value predicted by the linear relations found in [22]. This dramatically increases the efficiency of obtaining points fulfilling the $\delta m<0.1 \mathrm{GeV}$ requirement. We also checked that points outside the $2 \sigma$-band of the gaussian distribution never satisfy the mass splitting condition.

\subsection{Scan results}

For the results presented below we scan (with $14 \mathrm{k}$ points) over the ranges:

$$
\begin{aligned}
-42000 \mathrm{GeV} & <A_{0} \quad<-1000 \mathrm{GeV}, \\
630 \mathrm{GeV} & <M_{1 / 2}<1100 \mathrm{GeV} \\
\text { and } \quad 144 \mathrm{GeV} & <m_{0}<463 \mathrm{GeV}
\end{aligned}
$$

with $\tan \beta=10$ and $\mu>0$. The requirements on the stau-neutralino mass splitting drastically restricts the allowed CMSSM parameter space. In figure 6 we show the $m_{0^{-}}$ $M_{1 / 2}$ plane along with the corresponding values for the Higgs mass and $\Omega_{\widetilde{\chi}^{0}} h^{2}$. All the points shown satisfy $\delta m<0.1 \mathrm{GeV}$ and contain a neutralino LSP. As we can see, at the right edge of the points shown, the Higgs mass falls below $120 \mathrm{GeV}$, while for $M_{1 / 2} \gtrsim 1 \mathrm{TeV}$, the neutralino relic density violates Planck's upper bound. Furthermore, to the left of the points shown (low $m_{0}$ ), the stau becomes the LSP. We also show values for the stau relic abundance, computed according to eqs. (4.5) and (4.6). As we can see all points allow for the minimum stau yield required to provide a solution to the ${ }^{7} \mathrm{Li}$-problem. Finally, we show

\footnotetext{
${ }^{5}$ This loose interval on the Higgs mass window is due to the large theoretical uncertainties on the Higgs mass calculation in the MSSM. Furthermore, a more strict choice for the Higgs mass interval would not change our subsequent results.
} 

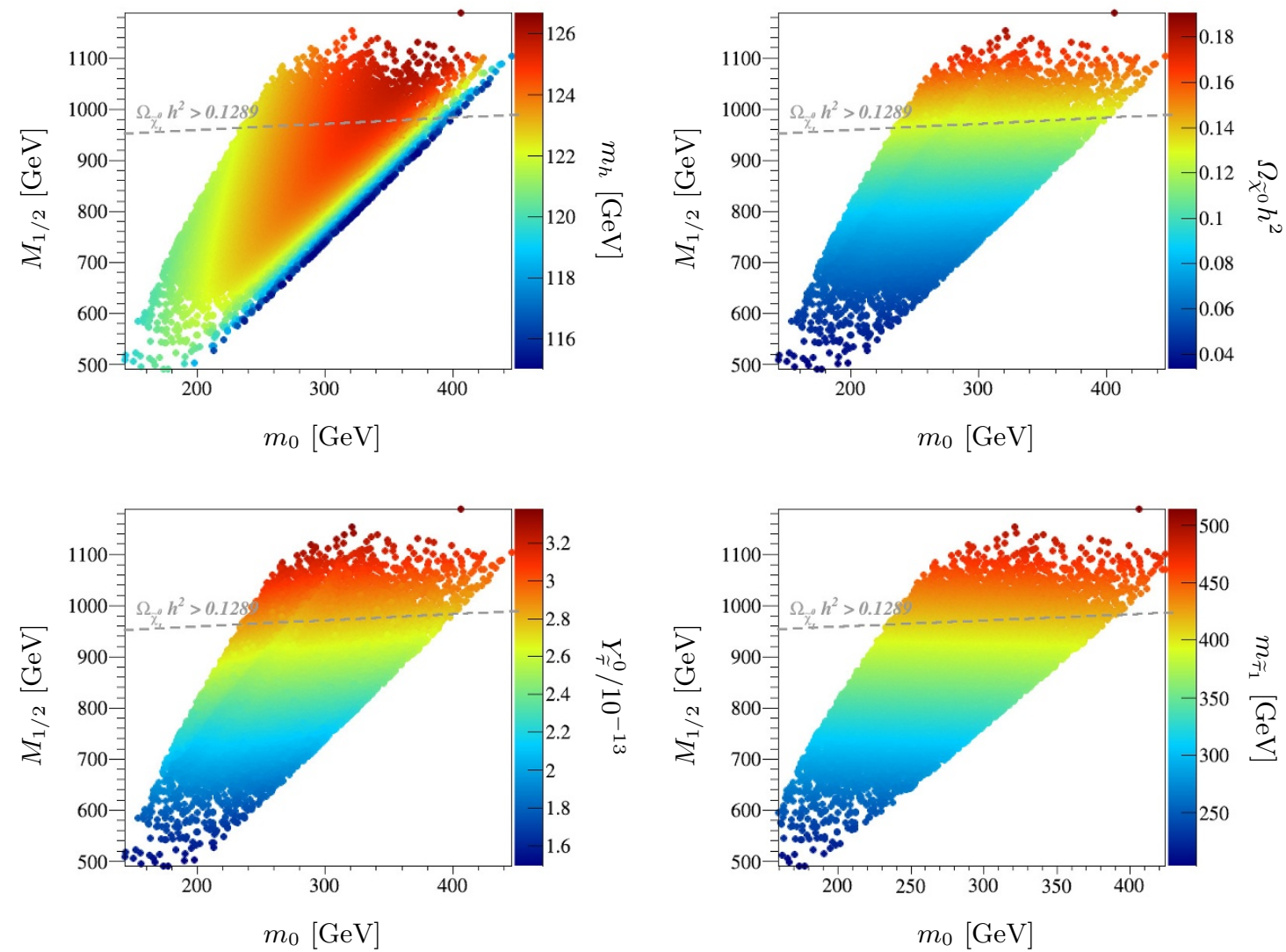

Figure 6. Values for the Higgs mass (top-left), the neutralino relic density (top-right) and the stau yield (bottom-left) in the $m_{0}-M_{1 / 2}$ plane, after requiring $\delta m<0.1 \mathrm{GeV}$ and a neutralino LSP. In the bottom-right plot we show values for the lightest stau mass $\left(m_{\widetilde{\tau}_{1}}\right)$ after the additional constraints on $m_{h}$ and $Y_{\widetilde{\tau}}^{0}$ have been imposed (see text for details).

in the bottom-right plot values for the lightest stau mass after imposing all the minimal conditions listed above. As shown, the constraints on the Higgs mass and the relic density (as well as $\delta m$ ) imply $200 \mathrm{GeV}<m_{\widetilde{\tau}_{1}}<460 \mathrm{GeV}$.

LHC constraints. After identifying the region of the CMSSM parameter space consistent with Planck $\left(\Omega_{\widetilde{\chi}^{0}} h^{2}<0.1283\right)$, the Higgs mass and the solution to the ${ }^{7} \mathrm{Li}$-problem we proceed to discuss the constraints from MET and HSCP searches at the LHC. As described in section 3, we have modified the public version of SMODELS to include the CMS search for HSCPs. Within this framework we can simultaneously apply the MET and HSCP constraints to the CMSSM parameter space. While the MET constraints directly make use of the upper limits on the production cross sections (for a given simplified model) provided by ATLAS and CMS, the HSCP constraints use the efficiency maps for the CMS exotic search [11], as described in section 2.2. Since the cascade decays of the SUSY particles in the scenario considered here may end up either on the lightest stau or on the lightest neutralino, we expect both the MET and the HSCP searches to be relevant for the parameter space shown in figure 6 . Therefore we simultaneously apply both constraints 

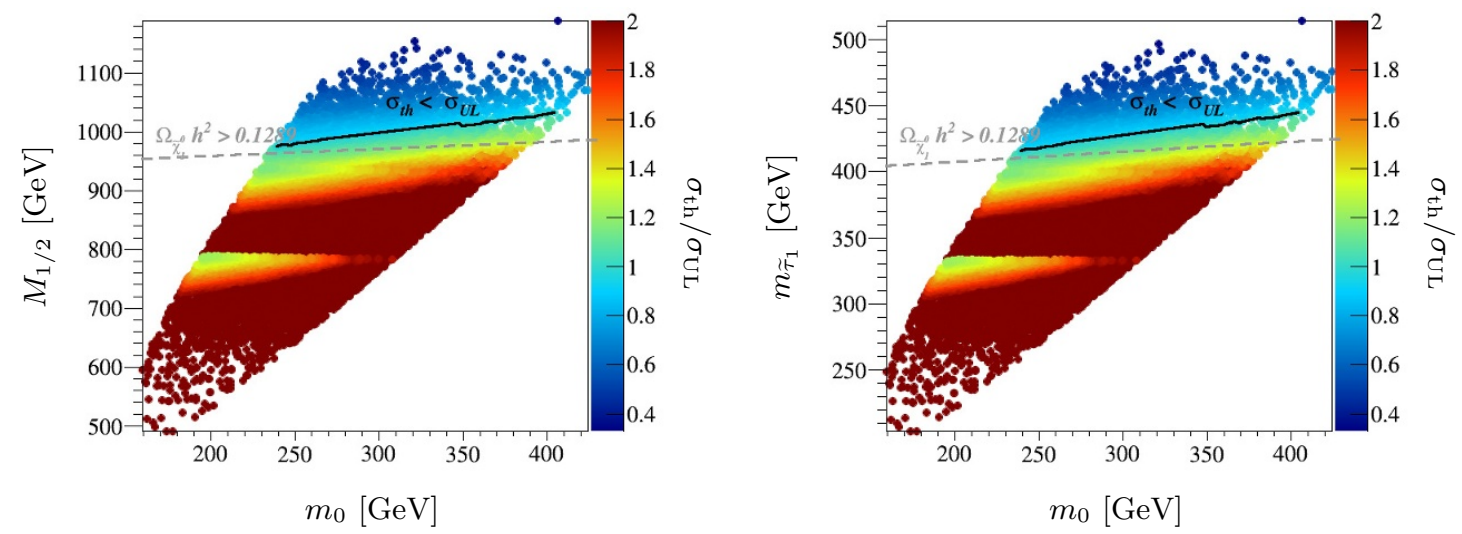

Figure 7. Values for the signal cross section $\left(\sigma_{\text {th }}\right)$ over the experimental 95\% CL upper limit $\left(\sigma_{\mathrm{UL}}\right)$ in the $m_{0}-M_{1 / 2}$ (left) and $m_{0}-m_{\widetilde{\tau}_{1}}$ (right) planes. Points with $\sigma_{\mathrm{th}} / \sigma_{\mathrm{UL}}>1$ are excluded by either MET searches or HSCP searches at the LHC.

and we consider a point in the parameter space excluded if, for at least one of the MET or the HSCP searches, the signal cross section for a given topology (or sum of topologies in the case of HSCP searches), $\sigma_{\mathrm{th}}$, is higher than its corresponding experimental upper limit $\left(\sigma_{\mathrm{UL}}\right)$.

In figure 7 we show the $\sigma_{\text {th }} / \sigma_{\mathrm{UL}}$ ratio $^{6}$ in the $m_{0}-M_{1 / 2}$ and $m_{0}-m_{\widetilde{\tau}_{1}}$ planes, as well as the lines for the upper limit on $\Omega_{\widetilde{\chi}^{0}} h^{2}$ (dashed gray) and $\sigma_{\mathrm{th}} / \sigma_{\mathrm{UL}}=1$ (solid black). Values up to $M_{1 / 2} \simeq 1 \mathrm{TeV}$ can be excluded, which corresponds to $m_{\widetilde{\tau}_{1}} \simeq 450 \mathrm{GeV}$. We also notice that the excluded region (where $\sigma_{\mathrm{th}} / \sigma_{\mathrm{UL}}>1$ ) extends to higher $M_{1 / 2}$ values when $m_{0}$ reaches its highest values (right edge). In this region the stop mass is suppressed due to large $\left|A_{0}\right|$ values and the pair production of stops is considerably enhanced, thus resulting in higher signal cross sections. The CMS paper [11] provides a conservative limit on the stau mass, $m_{\widetilde{\tau}_{1}}>260 \mathrm{GeV}$, which is based on exclusive stau pair production and is fairly model independent. Here, however, we can derive a much more stringent bound, since we are able to consider the inclusive production of staus from production of heavier sparticles and their decays. ${ }^{7}$

We can also compare the bound derived here with the one obtained by CMS for the inclusive stau production in the GMSB scenario (see ref. [11] for details). In the GMSB case the inclusive production (for a given $m_{\widetilde{\tau}_{1}}$ ) is smaller than in the CMSSM scenario discussed above, due to the presence of heavier squarks and gluinos. Nonetheless, CMS quotes $m_{\widetilde{\tau}_{1}}>500 \mathrm{GeV}$, which is higher than the one found here for the CMSSM scenario. This is mainly due to the fact that, while $100 \%$ of the GMSB signal considered by CMS goes to HSCP final states, a considerable fraction of our (CMSSM) signal goes into final states with missing energy (neutralino final states), thus reducing the reach of HSCP searches.

\footnotetext{
${ }^{6}$ Since here we are simultaneously considering HSCP and MET constraints, we only show the $\sigma_{\text {th }} / \sigma_{\mathrm{UL}}$ ratio for the most constraining analysis (maximum ratio).

${ }^{7}$ As already mentioned, since we only have computed the efficiencies for the finite number of simplified models listed in tables 1 and 2, all topologies not included in the $\mathcal{M} 1-\mathcal{M} 8$ models or falling outside our mass grid do not contribute to $\sigma_{\text {th }}$.
} 

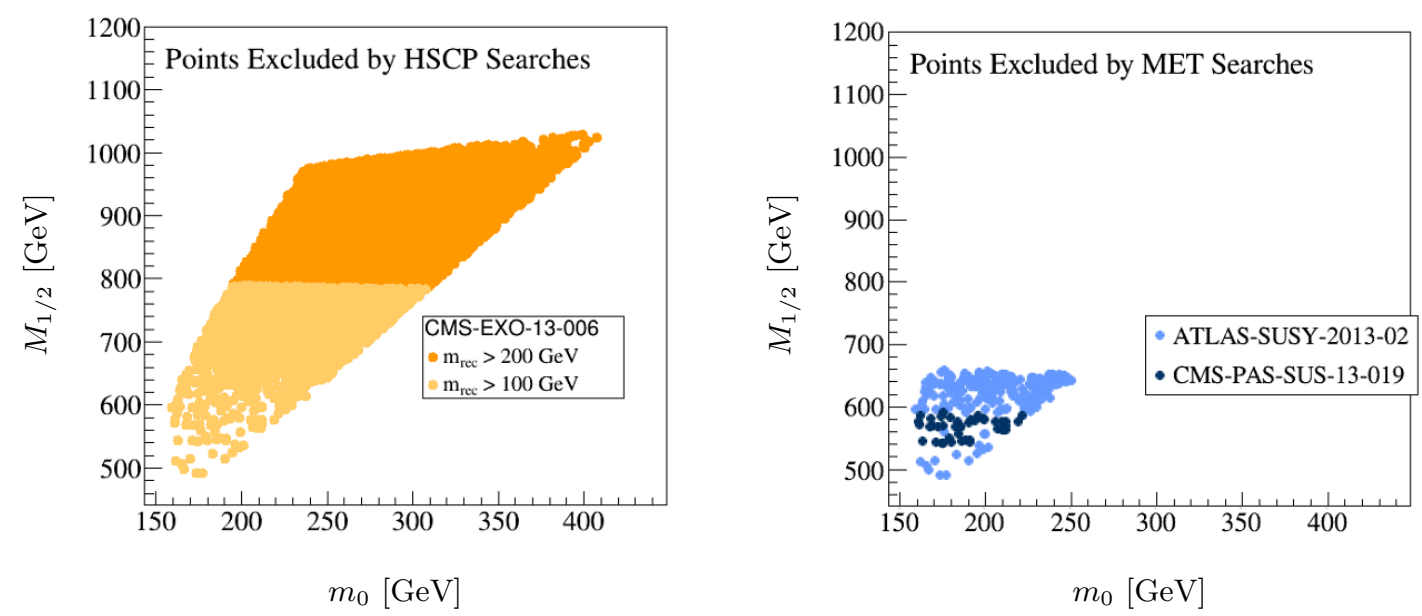

Figure 8. Points excluded at $95 \%$ CL by HSCP (left) and MET (right) searches in the $m_{0}-M_{1 / 2}$ plane. The distinct signal regions for the HSCP search from CMS-EXO-13-006 [11] are shown as light orange $\left(\mathrm{SR}_{100}\right)$ and dark orange $\left(\mathrm{SR}_{200}\right)$. Signal regions $\mathrm{SR}_{0}$ and $\mathrm{SR}_{300}$ were also considered but are less constraining than $\mathrm{SR}_{100}$ and $\mathrm{SR}_{200}$ for this model. For the MET searches we show by distinct colors the constraints from CMS [54] (dark blue) and ATLAS [55] (light blue) analyses.

Furthermore, the signal efficiencies for the events containing one or two HSCPs are smaller for the CMSSM than for the GMSB scenario, as a result of the different spectra as well as the fact that most of the events in the CMSSM signal contain only one HSCP. Nonetheless, we are still able to exclude all the region of parameter space (for $\tan \beta=10$ ) consistent with Planck's upper bound on the Dark Matter relic abundance. Therefore we conclude that, for low values of $\tan \beta$, the solution to the ${ }^{7} \mathrm{Li}$ within the CMSSM is no longer compatible with the LHC and Dark Matter constraints.

Finally, we comment on the feature appearing around $M_{1 / 2} \simeq 750 \mathrm{GeV}$ or $m_{\widetilde{\tau}_{1}} \simeq$ $320 \mathrm{GeV}$ in figure 7. As discussed in section 2.2, for the HSCP searches we consider four signal regions $\left(\mathrm{SR}_{0}, \mathrm{SR}_{100}, \mathrm{SR}_{200}\right.$ and $\left.\mathrm{SR}_{300}\right)$. For $m_{\widetilde{\tau}_{1}}<334 \mathrm{GeV}$ the efficiencies for $\mathrm{SR}_{200}$ and $\mathrm{SR}_{300}$ are taken as zero, so the parameter space is constrained by the upper limits for $\mathrm{SR}_{100}$. Once $m_{\widetilde{\tau}_{1}}>334 \mathrm{GeV}\left(M_{1 / 2}>800 \mathrm{GeV}\right)$, the efficiencies for $\mathrm{SR}_{200}$ are no longer zero and this signal region becomes the most constraining one, as shown by the sharp transition seen in figure 7 . This transition, however, does not affect our results, since all the points in this region are excluded.

Since in the model considered here the signal cross section splits into a HSCP signal and a MET signal, we expect both the MET and the HSCP searches to have a smaller reach than in a scenario where the signature is pure MET or pure HSCP. In order to compare the reach of MET searches against the one of HSCP searches, we show in figure 8 the most constraining (the one with the highest $\sigma_{\mathrm{th}} / \sigma_{\mathrm{UL}}$ ratio) HSCP analysis (left) and MET analysis (right). As we can see, the constraints from MET searches exclude points up to $M_{1 / 2} \simeq 650 \mathrm{GeV}$, which corresponds to $m_{\tilde{g}} \simeq 1500 \mathrm{GeV}$ or $m_{\tilde{q}} \simeq 1350 \mathrm{GeV}$. The most constraining topologies in this case are simply squark pair production followed by direct decay to the neutralino LSP. On the other hand, the HSCP searches can exclude up to 


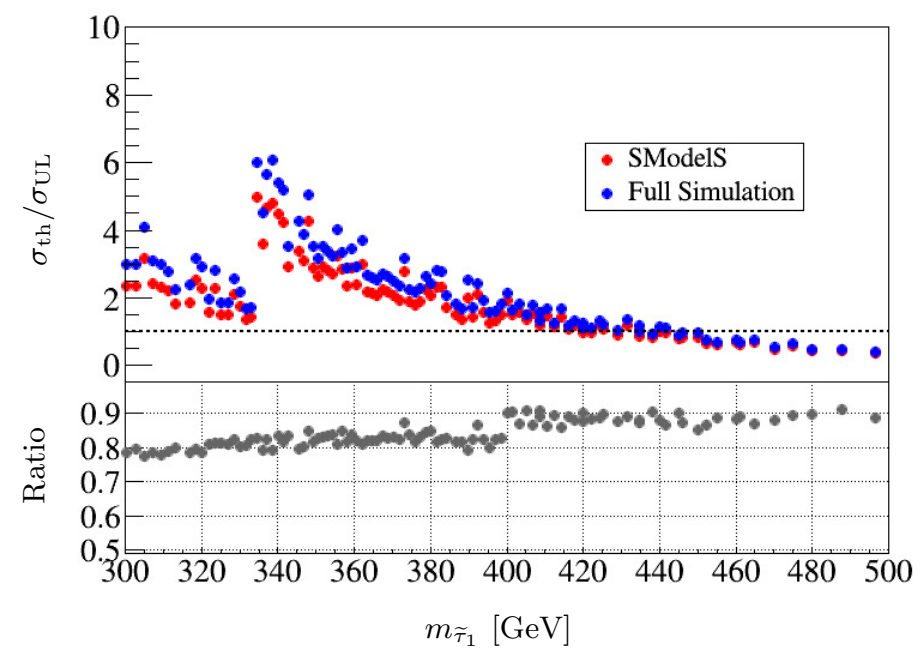

Figure 9. Comparison between the results obtained with the full simulation (blue) and the modified SMODELS version (red) for the HSCP search. In the upper frame we show the ratios between the signal cross section $\left(\sigma_{\mathrm{th}}\right)$ and the $95 \% \mathrm{CL}$ upper limit $\left(\sigma_{\mathrm{UL}}\right)$ for the two methods, whilst the lower frame shows their ratio, i.e. $\left(\sigma_{\mathrm{th}} / \sigma_{\mathrm{UL}}\right)_{\mathrm{Full}} /\left(\sigma_{\mathrm{th}} / \sigma_{\mathrm{UL}}\right)_{\mathrm{SModelS}}$.

$M_{1 / 2}=1 \mathrm{TeV}$ or $m_{\tilde{g}}=2250 \mathrm{GeV}$ and $m_{\tilde{q}}=2050 \mathrm{GeV}$. As expected, the HSCP constraints allows us to exclude a much larger region of the parameter space.

\subsection{Comparison with full simulation}

As already mentioned, the method outlined in section 3 used to obtain the results above relies on a few approximations. First, several details of the full model are neglected, such as the spin of the intermediate particles, the production channel ( $t$-channel or $s$-channel), kinematical effects due to off-shell decays and others. Second, when computing the signal cross sections $\left(\sigma_{\text {th }}\right)$ for the full model we can only include the simplified models $\mathcal{M} 1-\mathcal{M} 8$ for which we have computed efficiencies. Finally other small effects such as the interpolation of the efficiency map grid and the effect of neglecting topologies with weights below $\tilde{\sigma}_{\text {min }}$ can also affect the final result obtained through the simplified models approach. Therefore it is relevant to compare the results obtained in section 4.1 with a full Monte Carlo simulation. In order to make this comparison we select $\mathcal{O}(100)$ representative points from the CMSSM scan discussed in section 4.1 and compute their signal cross sections for each of the HSCP signal regions performing the full simulation via PYTHIA 6 followed by the analysis detailed in section 2.2 .

In figure 9 we show the ratio $\sigma_{\mathrm{th}} / \sigma_{\mathrm{UL}}$ for the best signal region as a function of the stau mass obtained from the modified SModelS version (red points) and from the full simulation (blue points). The lower frame shows the ratio between $\sigma_{\mathrm{th}} / \sigma_{\mathrm{UL}}$ for the full simulation and SModelS. As shown in the figure, the agreement is within $\sim 20 \%$ for all of the mass range shown. As already mentioned, since we do not compute efficiencies for all possible simplified models, we expect the excluded region obtained by SMODELS to be conservative. This is indeed what is seen in figure 9, where the SMODELS value for $\sigma_{\text {th }}$ is always below the one obtained with the full simulation. In order to guide the eye we also 
show $\sigma_{\mathrm{th}} / \sigma_{\mathrm{UL}}=1$ as a dashed line, hence all points above this line are excluded. Even though SMOdELS is conservative, both SMODELS and the full simulation exclude stau masses up to $\simeq 450 \mathrm{GeV}$, as already found in section 4.1 .

\section{Conclusions}

Heavy stable charged particles (HSCPs) provide a prominent signature at the LHC and are present in several well motivated BSM scenarios. Most of the current experimental searches for HSCPs present their results for specific BSM models, thus making it difficult to apply them to other scenarios of interest. Here we have re-interpreted these results in terms of simplified models, what allowed us to derive constraints to a wide range of arbitrary BSM models not considered previously. To this end we have presented a new method to systematically decompose full BSM models as a coherent sum of simplified models containing both stable and quasi-stable particles. To this end we have defined a set of eight simplified models containing one or two HSCPs in the final states and computed the corresponding signal efficiencies as a function of the model parameters. With the inclusion of both the new decomposition method and the efficiency maps to the program package SMODELS we are able to apply both MET and HSCP constraints to arbitrary BSM models containing a $\mathbb{Z}_{2}$ symmetry.

We showed that HSCP constraints on full BSM models can be reliably applied through the simplified model framework presented here. The constraints obtained by SMODELS on the signal cross-sections for the scenario studied here agreed within $\sim 20 \%$ with the full Monte Carlo simulation. These differences are similar to other theoretical uncertainties and, when translated to constraints on the sparticles masses, do not lead to any significant difference between the full simulation and SMODELS results. Therefore we conclude that the simplified models introduced here along with the SModeLS tools are well suited for confronting full models with experimental HSCP searches.

We then applied our modified SModELS program to the CMSSM stau co-annihilation strip, particularly considering the case of a nearly mass degenerate stau and neutralino. In this part of the parameter space the stau becomes long-lived providing the HSCP signature and presenting a potential solution to the Lithium problem. As the decay chains following the production of heavier SUSY particles can terminate in either the neutralino or the stau, we encounter in this scenario both MET and HSCP signatures. We have shown that the MET constraints for single simplified model topologies allow us to exclude points up to $m_{\widetilde{\tau}_{1}}=275 \mathrm{GeV}$ ( or $m_{\tilde{g}} \simeq 1500 \mathrm{GeV}$ and $m_{\tilde{q}} \simeq 1350 \mathrm{GeV}$ ). On the other hand, using the efficiency maps computed here, the HSCP searches can exclude up to $m_{\widetilde{\tau}_{1}}=450 \mathrm{GeV}$ (or $m_{\tilde{g}}=2250 \mathrm{GeV}$ and $\left.m_{\tilde{q}}=2050 \mathrm{GeV}\right)$. For small $\tan \beta$ values, the HSCP searches exclude the whole parameter space consistent with a potential solution to the Lithium problem and Planck's bound on the neutralino relic abundance. 


\section{Acknowledgments}

We are grateful to the SModelS group for useful discussions. This work was supported by the German Federal Ministry of Education and Research (BMBF), by Fundação de Amparo à Pesquisa do Estado de São Paulo (FAPESP) and by the Fonds de la Recherche Scientifique (FNRS).

Open Access. This article is distributed under the terms of the Creative Commons Attribution License (CC-BY 4.0), which permits any use, distribution and reproduction in any medium, provided the original author(s) and source are credited.

\section{References}

[1] P.F. Smith et al., A search for anomalous hydrogen in enriched $D_{2} O$, using a time-of-flight spectrometer, Nucl. Phys. B 206 (1982) 333 [INSPIRE].

[2] T.K. Hemmick et al., A search for anomalously heavy isotopes of low $Z$ nuclei, Phys. Rev. D 41 (1990) 2074 [INSPIRE].

[3] T. Yamagata, Y. Takamori and H. Utsunomiya, Search for anomalously heavy hydrogen in deep sea water at $4000 \mathrm{~m}$, Phys. Rev. D 47 (1993) 1231 [INSPIRE].

[4] N.E. Bomark, A. Kvellestad, S. Lola, P. Osland and A.R. Raklev, Long lived charginos in natural SUSY?, JHEP 05 (2014) 007 [arXiv:1310.2788] [INSPIRE].

[5] M. Byrne, Universal extra dimensions and charged LKPs, Phys. Lett. B 583 (2004) 309 [hep-ph/0311160] [INSPIRE].

[6] T. Jittoh et al., Possible solution to the ${ }^{7}$ Li problem by the long lived stau, Phys. Rev. D 76 (2007) 125023 [arXiv:0704.2914] [INSPIRE].

[7] T. Jittoh, J. Sato, T. Shimomura and M. Yamanaka, Long life stau in the minimal supersymmetric standard model, Phys. Rev. D 73 (2006) 055009 [Erratum ibid. D 87 (2013) 019901] [hep-ph/0512197] [INSPIRE].

[8] H. Pagels and J.R. Primack, Supersymmetry, cosmology and new TeV physics, Phys. Rev. Lett. 48 (1982) 223 [INSPIRE].

[9] L. Covi, J.E. Kim and L. Roszkowski, Axinos as cold dark matter, Phys. Rev. Lett. 82 (1999) 4180 [hep-ph/9905212] [INSPIRE].

[10] CMS collaboration, Searches for long-lived charged particles in pp collisions at $\sqrt{s}=7$ and $8 \mathrm{TeV}$, JHEP 07 (2013) 122 [arXiv:1305.0491] [INSPIRE].

[11] CMS collaboration, Constraints on the pMSSM, AMSB model and on other models from the search for long-lived charged particles in proton-proton collisions at $\sqrt{s}=8 \mathrm{TeV}$, Eur. Phys. J. C 75 (2015) 325 [arXiv: 1502.02522] [INSPIRE].

[12] ATLAS collaboration, Searches for heavy long-lived charged particles with the ATLAS detector in proton-proton collisions at $\sqrt{s}=8 \mathrm{TeV}$, JHEP 01 (2015) 068 [arXiv:1411.6795] [INSPIRE].

[13] J. Alwall, P. Schuster and N. Toro, Simplified models for a first characterization of new physics at the LHC, Phys. Rev. D 79 (2009) 075020 [arXiv:0810.3921] [InSPIRE]. 
[14] LHC New Physics Working Group collaboration, D. Alves, Simplified models for LHC new physics searches, J. Phys. G 39 (2012) 105005 [arXiv:1105.2838] [INSPIRE].

[15] ATLAS collaboration, H. Okawa, Interpretations of SUSY searches in ATLAS with simplified models, in Proceedings of Particles and fields. Meeting of the Division of the American Physical Society, Providence U.S.A., 9-13 Aug 2011 [arXiv:1110.0282] [INSPIRE].

[16] CMS collaboration, Interpretation of searches for supersymmetry with simplified models, Phys. Rev. D 88 (2013) 052017 [arXiv:1301.2175] [INSPIRE].

[17] S. Kraml et al., SModelS: a tool for interpreting simplified-model results from the LHC and its application to supersymmetry, Eur. Phys. J. C 74 (2014) 2868 [arXiv:1312.4175] [INSPIRE].

[18] M. Papucci, K. Sakurai, A. Weiler and L. Zeune, Fastlim: a fast LHC limit calculator, Eur. Phys. J. C 74 (2014) 3163 [arXiv:1402.0492] [InSPIRE].

[19] J. Heisig and J. Kersten, Long-lived staus from strong production in a simplified model approach, Phys. Rev. D 86 (2012) 055020 [arXiv:1203.1581] [INSPIRE].

[20] J. Heisig, J. Kersten, B. Panes and T. Robens, A survey for low stau yields in the MSSM, JHEP 04 (2014) 053 [arXiv:1310.2825] [INSPIRE].

[21] S. Kraml et al., SModelS v1.0: a short user guide, arXiv:1412.1745 [INSPIRE].

[22] Y. Konishi et al., First evidence of the constrained minimal supersymmetric standard model is appearing soon, Phys. Rev. D 89 (2014) 075006 [arXiv: 1309.2067] [INSPIRE].

[23] M. Citron et al., End of the CMSSM coannihilation strip is nigh, Phys. Rev. D 87 (2013) 036012 [arXiv:1212.2886] [INSPIRE].

[24] N. Desai, J. Ellis, F. Luo and J. Marrouche, Closing in on the tip of the CMSSM stau coannihilation strip, Phys. Rev. D 90 (2014) 055031 [arXiv:1404.5061] [INSPIRE].

[25] J. Alwall, M. Herquet, F. Maltoni, O. Mattelaer and T. Stelzer, MadGraph 5: going beyond, JHEP 06 (2011) 128 [arXiv:1106.0522] [INSPIRE].

[26] T. Sjöstrand, S. Mrenna and P.Z. Skands, PYTHIA 6.4 physics and manual, JHEP 05 (2006) 026 [hep-ph/0603175] [INSPIRE].

[27] A. Buckley, PySLHA: a Pythonic interface to SUSY Les Houches Accord data, Eur. Phys. J. C 75 (2015) 467 [arXiv: 1305.4194] [INSPIRE].

[28] F. Spite and M. Spite, Abundance of lithium in unevolved halo stars and old disk stars: interpretation and consequences, Astron. Astrophys. 115 (1982) 357 [INSPIRE].

[29] R.H. Cyburt, B.D. Fields and K.A. Olive, An update on the big bang nucleosynthesis prediction for ${ }^{7}$ Li: the problem worsens, JCAP 11 (2008) 012 [arXiv:0808.2818] [INSPIRE].

[30] R.H. Cyburt, B.D. Fields, K.A. Olive and T.-H. Yeh, Big bang nucleosynthesis: 2015, arXiv: 1505.01076 [INSPIRE].

[31] L. Sbordone et al., The metal-poor end of the Spite plateau. I. Stellar parameters, metallicities and lithium abundances, Astron. Astrophys. 522 (2010) A26 [arXiv:1003.4510] [INSPIRE].

[32] R.N. Boyd, C.R. Brune, G.M. Fuller and C.J. Smith, New nuclear physics for big bang nucleosynthesis, Phys. Rev. D 82 (2010) 105005 [arXiv: 1008.0848] [INSPIRE].

[33] K. Jedamzik, Did something decay, evaporate, or annihilate during big bang nucleosynthesis?, Phys. Rev. D 70 (2004) 063524 [astro-ph/0402344] [INSPIRE]. 
[34] K. Jedamzik, K.-Y. Choi, L. Roszkowski and R. Ruiz de Austri, Solving the cosmic lithium problems with gravitino dark matter in the CMSSM, JCAP 07 (2006) 007 [hep-ph/0512044] [INSPIRE].

[35] M. Pospelov, Particle physics catalysis of thermal big bang nucleosynthesis, Phys. Rev. Lett. 98 (2007) 231301 [hep-ph/0605215] [INSPIRE].

[36] R.H. Cyburt, J.R. Ellis, B.D. Fields, K.A. Olive and V.C. Spanos, Bound-state effects on light-element abundances in gravitino dark matter scenarios, JCAP 11 (2006) 014 [astro-ph/0608562] [INSPIRE].

[37] K. Jedamzik, The cosmic ${ }^{6} L i$ and ${ }^{7}$ Li problems and BBN with long-lived charged massive particles, Phys. Rev. D 77 (2008) 063524 [arXiv:0707.2070] [INSPIRE].

[38] M. Kusakabe, T. Kajino, R.N. Boyd, T. Yoshida and G.J. Mathews, A simultaneous solution to the ${ }^{6} \mathrm{Li}$ and ${ }^{7} \mathrm{Li}$ big bang nucleosynthesis problems from a long-lived negatively-charged leptonic particle, Phys. Rev. D 76 (2007) 121302 [arXiv:0711.3854] [INSPIRE].

[39] T. Jittoh et al., Big-bang nucleosynthesis and the relic abundance of dark matter in a stau-neutralino coannihilation scenario, Phys. Rev. D 78 (2008) 055007 [arXiv:0805.3389] [INSPIRE].

[40] M. Pospelov, J. Pradler and F.D. Steffen, Constraints on supersymmetric models from catalytic primordial nucleosynthesis of beryllium, JCAP 11 (2008) 020 [arXiv:0807.4287] [INSPIRE].

[41] T. Jittoh et al., Stau relic density at the big-bang nucleosynthesis era consistent with the abundance of the light element nuclei in the coannihilation scenario, Phys. Rev. D 82 (2010) 115030 [arXiv: 1001.1217] [INSPIRE].

[42] R.H. Cyburt et al., Metastable charged sparticles and the cosmological ${ }^{7}$ Li problem, JCAP 12 (2012) 037 [arXiv: 1209.1347] [INSPIRE].

[43] M. Kusakabe et al., Revised big bang nucleosynthesis with long-lived negatively charged massive particles: updated recombination rates, primordial ${ }^{9}$ Be nucleosynthesis and impact of new ${ }^{6}$ Li limits, Astrophys. J. Suppl. 214 (2014) 5 [arXiv:1403.4156] [INSPIRE].

[44] W. Porod and F. Staub, SPheno 3.1: extensions including flavour, CP-phases and models beyond the MSSM, Comput. Phys. Commun. 183 (2012) 2458 [arXiv:1104.1573] [INSPIRE].

[45] W. Beenakker, R. Hopker, M. Spira and P.M. Zerwas, Squark and gluino production at hadron colliders, Nucl. Phys. B 492 (1997) 51 [hep-ph/9610490] [INSPIRE].

[46] W. Beenakker, M. Krämer, T. Plehn, M. Spira and P.M. Zerwas, Stop production at hadron colliders, Nucl. Phys. B 515 (1998) 3 [hep-ph/9710451] [INSPIRE].

[47] A. Kulesza and L. Motyka, Threshold resummation for squark-antisquark and gluino-pair production at the LHC, Phys. Rev. Lett. 102 (2009) 111802 [arXiv:0807.2405] [INSPIRE].

[48] A. Kulesza and L. Motyka, Soft gluon resummation for the production of gluino-gluino and squark-antisquark pairs at the LHC, Phys. Rev. D 80 (2009) 095004 [arXiv:0905.4749] [INSPIRE].

[49] W. Beenakker et al., Soft-gluon resummation for squark and gluino hadroproduction, JHEP 12 (2009) 041 [arXiv:0909.4418] [INSPIRE].

[50] W. Beenakker et al., Supersymmetric top and bottom squark production at hadron colliders, JHEP 08 (2010) 098 [arXiv: 1006.4771] [INSPIRE]. 
[51] W. Beenakker et al., Squark and gluino hadroproduction, Int. J. Mod. Phys. A 26 (2011) 2637 [arXiv:1105.1110] [InSPIRE].

[52] G. Bélanger, F. Boudjema, A. Pukhov and A. Semenov, MicrOMEGAs_3: a program for calculating dark matter observables, Comput. Phys. Commun. 185 (2014) 960 [arXiv: 1305.0237] [INSPIRE].

[53] Planck collaboration, P.A.R. Ade et al., Planck 2013 results. XVI. Cosmological parameters, Astron. Astrophys. 571 (2014) A16 [arXiv:1303.5076] [INSPIRE].

[54] CMS collaboration, Searches for supersymmetry using the $M_{T 2}$ variable in hadronic events produced in pp collisions at $8 \mathrm{TeV}$, JHEP 05 (2015) 078 [arXiv: 1502.04358] [INSPIRE].

[55] ATLAS collaboration, Search for squarks and gluinos with the ATLAS detector in final states with jets and missing transverse momentum using $\sqrt{s}=8 \mathrm{TeV}$ proton-proton collision data, JHEP 09 (2014) 176 [arXiv: 1405.7875] [INSPIRE]. 